\title{
Computation and numerical simulation of focused undulator radiation for optical stochastic cooling
}

\author{
M. B. Andorf, ${ }^{1}$ V. A. Lebedev, ${ }^{2}$ J. Jarvis, ${ }^{2}$ and P. Piot ${ }^{1,2}$ \\ ${ }^{1}$ Northern Illinois Center for Accelerator \& Detector Development and Department of Physics, \\ Northern Illinois University, DeKalb, Illinois 60115, USA \\ ${ }^{2}$ Fermi National Accelerator Laboratory, Batavia, Illinois 60510, USA
}

(Received 14 June 2018; published 19 October 2018)

\begin{abstract}
Optical stochastic cooling (OSC) is a promising technique for the cooling of dense particle beams. Its operation at optical frequencies enables obtaining a much larger bandwidth compared to the well-known microwave-based stochastic cooling. In the OSC undulator radiation generated by a particle in an upstream "pickup" undulator is amplified and focused at the location of a downstream "kicker" undulator. Inside the kicker, a particle interacts with its own radiation field from the pickup. The resulting interaction produces a longitudinal kick with its value depending on the particles momentum which, when correctly phased, yields longitudinal cooling. The horizontal cooling is achieved by introducing a coupling between longitudinal and horizontal degrees of freedom. Vertical cooling is achieved by coupling between horizontal and vertical motions in the ring. In this paper, we present formulas for computation of the corrective kick and validate them against numerical simulations performed with a wave-optics computer program.
\end{abstract}

DOI: 10.1103/PhysRevAccelBeams.21.100702

\section{INTRODUCTION}

Optical stochastic cooling (OSC) is a method of beam cooling in an accelerator that utilizes the short, radiation wave packet generated by a particle passing through an undulator, as a way to make a corrective kick in energy with minimal interference from other nearby particles in the beam. The method is an extension of the well-known microwave stochastic cooling [1-3] to optical wavelengths. A transition from microwave to optical frequencies enables an increase of the cooling bandwidth by 3-4 orders of magnitude. For a system operating at the optimal gain that shortens the cooling time in the same proportion [4-6].

In OSC the corrective kick occurs via interaction of a particle with its own radiation, radiated at an earlier time in a "pickup" undulator, imaged in a downstream identical undulator referred to as the "kicker"; See Fig. 1. The amount of energy exchange between the particle and its radiation is determined by the arrival time of the particle with respect to the radiation wave packet. This difference in arrival time depends on the particles phase-space coordinates and the lattice optics associated with the bypass beamline (typically a magnetic chicane) inserted between the pickup and kicker. For a properly tuned chicane

Published by the American Physical Society under the terms of the Creative Commons Attribution 4.0 International license. Further distribution of this work must maintain attribution to the author(s) and the published article's title, journal citation, and DOI. averaging kicks over many turns simultaneously provides cooling in the horizontal and longitudinal planes. Although the kick is corrective in energy only; the coupling between longitudinal and horizontal motions introduced in the beamline between pickup and kicker provides cooling in the horizontal plane. The coupling is achieved by appropriate choice of pickup-to-kicker transfer matrix and the introduction of dispersion in both undulators.

Despite its great promise to help in storage of high intensity beams [7], and in cooling of different types of beams [8-10], OSC has so far not been experimentally demonstrated. A proof-of-principle experiment is in preparation at Fermilab [11] using a $100-\mathrm{MeV}$ electron beam stored in the integrable-optics test accelerator (IOTA), a small-circumference ring dedicated to address issues pertaining to high-intensity beams. The experiment will be performed in two phases. The first phase will implement a "passive" version of the OSC technique where the radiation from the pickup undulator will be directly imaged to

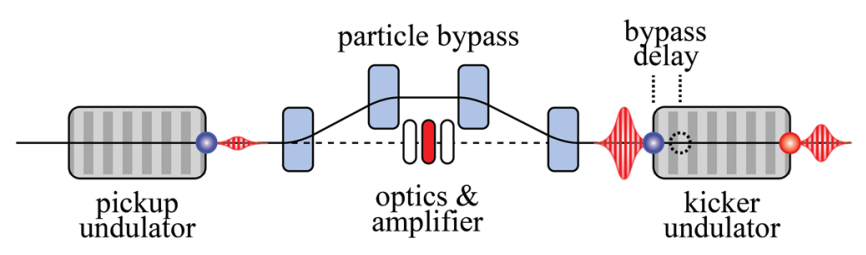

FIG. 1. Conceptual diagram of a transit-time OSC insertion. The blue circles and red wiggles respectively represent the charged-particle beam and radiation pulse. The beam passes through a by-pass beamline made of four dipole magnets (blue rectangles) and other (not shown) focusing elements. 
TABLE I. Undulator parameters for the OSC test planned at the IOTA facility with a $100-\mathrm{MeV}$ electron beam.

\begin{tabular}{lll}
\hline \hline Parameter, symbol & Value & Units \\
\hline Undulator parameter, $K$ & 1.038 & $\ldots$ \\
Length, $L_{u}$ & 77.4 & $\mathrm{~cm}$ \\
Undulator period, $\lambda_{u}$ & 11.06 & $\mathrm{~cm}$ \\
Number of periods, $N_{u}$ & 7 & $\ldots$ \\
On-axis wavelength, $\lambda_{o}$ & 2.2 & $\mu \mathrm{m}$ \\
Electron Lorentz factor, $\gamma$ & 195.69 & $\cdots$ \\
\hline \hline
\end{tabular}

the kicker undulator without any amplification [11,12]. A following phase will implement an optical amplifier to fully realize the potential of the OSC method.

In this paper, we present a semianalytical theory to compute the energy kick which a particle receives passing through an OSC system. We apply this theory to the proofof-principle test of the OSC to be carried out in the IOTA ring at Fermilab [13]. We should however stress that the theory is general enough to apply to any OSC system. The theoretical results are validated against wave-optics numerical simulations performed with the SYNCHROTRON RADIATION WORKSHOP (SRW) [14] program. The SRW program which is based on the principle of physical optics uses Fourier-optics methods to simulate wave propagation $[15,16]$. The program also includes a Liénard-Wiechert solver capable of modeling the radiation emitted as an electron propagates in a given magnetostatic field. SRW was successfully used to model the optical system associated to OSC. It took into account the realistic properties of the emitted radiation from the pickup; see Ref. [17] for the implementation. Table I lists undulator and electronbeam parameters considered throughout this paper. These values correspond to the planned OSC experiment at IOTA. The spectrum of the undulator radiation appears in Fig. 2. It also shows the angle-integrated full-width-halfmaximum relative bandwidth is on the order of $\sim 30 \%$.

\section{ELECTRIC FIELD AMPLITUDE IN THE KICKER UNDULATOR}

To find the OSC cooling rates we compute the on-axis electric field of the radiation focused to the kicker undulator. Below, if not stated otherwise, we imply that there is no optical amplifier present. As a major simplification we mitigate depth-of-field effects by locating the undulator such that their centers are at a distance $2 R_{o} \gg L_{u}$ apart where $L_{u}$ is the length of one undulator (the pickup and kicker undulator are taken to be identical). In this way we effectively only describe radiation which has emerged from

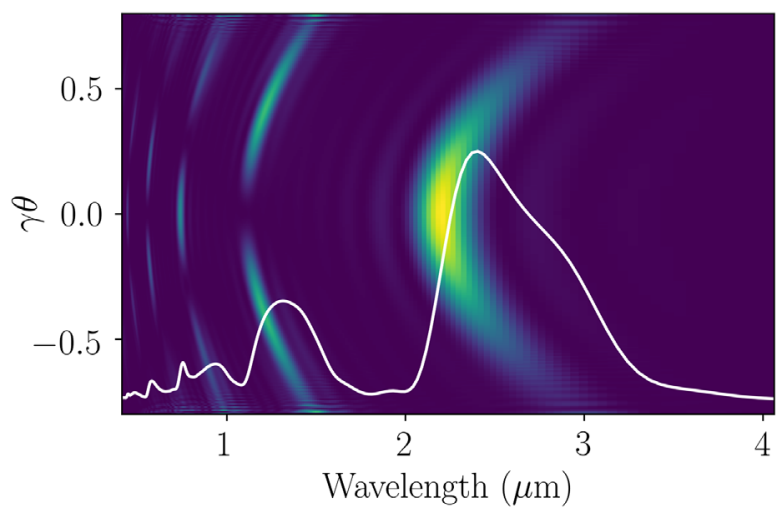

FIG. 2. Undulator radiation spectrum in the horizontal plane computed with SRW assuming the parameters given in Table I. The white trace integrates the spectrum in the transverse plane over an angular acceptance corresponding to $\gamma \theta_{m}=0.8$.

the pick-up center. A single focusing lens is placed symmetrically between the undulators with its focal length chosen to be $R_{o} / 2$. The problem is split in the following way: first we find the electric field on the lens surface in the time domain and expand it in a Fourier series. We then apply a modified version of Kirchoff's diffraction formula for a lens in order to find the field in the kicker before finally using the field amplitude to compute the longitudinal energy kick to the particle.

The electric field at the lens surface is found by using the Liénard-Wiechert formula (in the far-field zone)

$$
\boldsymbol{E}(r, t)=\frac{e}{c^{2}} \frac{(\boldsymbol{R}-\boldsymbol{\beta} R)(\boldsymbol{a} \cdot \boldsymbol{R})-\boldsymbol{a} R[R-(\boldsymbol{\beta} \cdot \boldsymbol{R})]}{(R-(\boldsymbol{\beta} \cdot \boldsymbol{R}))^{3}}
$$

where $e$ is the particle charge, $\boldsymbol{\beta}=\boldsymbol{v} / c, \boldsymbol{R}=\boldsymbol{r}-\boldsymbol{r}^{\prime}$ is a vector from the point of emitted radiation $r^{\prime}$ to the observation point $\boldsymbol{r}$ on the lens surface and $\boldsymbol{a}$ is the acceleration. All values are taken at the emitter (retarded) time $t^{\prime}=t-R_{o} / c$. The particle velocity components are

$$
\begin{aligned}
& v_{x}\left(t^{\prime}\right)=-c \theta_{e} \sin \left(\omega_{u} t^{\prime}\right), \quad \text { and } \\
& v_{z}\left(t^{\prime}\right)=c\left[1-\frac{1}{2 \gamma^{2}}-\frac{\theta_{e}^{2}}{2} \sin ^{2}\left(\omega_{u} t^{\prime}\right)\right],
\end{aligned}
$$

where $\omega_{u} \equiv c k_{u}$ is the undulator frequency and $\theta_{e}=K / \gamma$ is the amplitude of particle angle oscillation with $K \equiv$ $q B_{o} / k_{u} m c^{2}$ being the undulator strength parameter. Plugging Eq. (2) into Eq. (1) and expliciting $\boldsymbol{R}$ in the Cartesian-coordinate system, i.e., $\boldsymbol{R}=R_{o}[\sin (\theta) \cos (\phi) \hat{\boldsymbol{x}}+$ $\sin (\theta) \sin (\phi) \hat{\boldsymbol{y}}+\cos (\theta) \hat{z}]$, yields the horizontal component of the electric field at the lens

$$
E_{x}(r, t)=4 e \omega_{u} \gamma^{4} \cos \left(t^{\prime} \omega_{u}\right) \frac{1+\gamma^{2}\left[\theta^{2}\left(1-2 \cos ^{2}(\phi)\right)-2 \theta \theta_{e} \sin \left(t^{\prime} \omega_{u}\right) \cos (\phi)-\theta_{e}^{2} \sin \left(t^{\prime} \omega_{u}\right)\right]}{c R_{o}\left\{1+\gamma^{2}\left[\theta^{2}+2 \theta \theta_{e} \sin \left(t^{\prime} \omega_{u}\right) \cos (\phi)+\theta_{e}^{2} \sin ^{2}\left(t^{\prime} \omega_{u}\right)\right]\right\}}
$$

for angles $\theta \ll 1$. 
Additionally, and given that only the first harmonic interacts resonantly with the particle in the kicker, we ignore the effect of higher harmonics and relate the observation angle to the radiation frequency via

$$
\omega(\theta)=\frac{2 \gamma^{2} \omega_{u}}{1+\gamma^{2}\left(\theta^{2}+\theta_{e}^{2} / 2\right)} .
$$

To find the electric field in the kicker where the radiation has been focused we use a modified Kirchoff's formula ${ }^{1}$

$$
E\left(\boldsymbol{r}^{\prime \prime}\right)=\frac{1}{2 \pi i c} \int_{\Sigma} \frac{\omega(\theta) E_{w}(\boldsymbol{r})}{\left|\boldsymbol{r}-\boldsymbol{r}^{\prime \prime}\right|} e^{i \omega(\theta)\left|\boldsymbol{r}-\boldsymbol{r}^{\prime \prime}\right| / c} d \Sigma
$$

where $E_{\omega}(\boldsymbol{r})$ is the complex amplitude of the first harmonic at a given point on the lens surface

$$
E_{\omega}(\boldsymbol{r})=\frac{\omega(\theta)}{\pi} \int_{0}^{2 \pi / \omega(\theta)} E_{x}(r, t) e^{-i \omega(\theta) t} d t .
$$

The integration domain $\Sigma$ in Eq. (5) is the surface of the lens where the vector $\boldsymbol{r}$ is located, and $\boldsymbol{r}^{\prime \prime}$ is the coordinate of observation in the kicker. In the absence of dispersion in the lens a ray leaving the pickup with an angle $\theta$ will have a path lengthening equal to $2\left(R_{o} \theta^{2} / 2\right)$ which is exactly compensated by a decrease in the glass thickness of the lens such that all rays take the same amount of time to travel from pickup to kicker centers. ${ }^{2}$ This implies that the argument in the exponential of Eq. (5) reduces to a complex constant and can be dropped. Integrating Eq. (2) one obtains an expression for $R\left(t^{\prime}\right)$ which can be rearranged to give the observer time as a function of the emitter time

$$
\begin{aligned}
t\left(t^{\prime}\right)= & \frac{R_{o}}{c}\left(1+\frac{\theta^{2}}{2}\right)+\frac{t^{\prime}}{2 \gamma^{2}}\left[1+\gamma^{2}\left(\theta^{2}+\frac{\theta_{e}^{2}}{2}\right)\right] \\
& -\frac{\theta_{e}^{2}}{8 \omega_{u}} \sin \left(2 \omega_{u} t^{\prime}\right)-\frac{\theta \theta_{e}}{\omega_{u}} \cos (\phi) \cos \left(\omega_{u} t^{\prime}\right) .
\end{aligned}
$$

Computing the derivative $d t / d t^{\prime}$ together with using Eqs. (3) and (7) enables integration of Eq. (5).

\footnotetext{
${ }^{1}$ This is an approximate formula which however yields the exact result for the case of small undulator parameter and infinite angular acceptance of the focusing system. The exact solution would require a multidimensional Fourier transform of the incoming wave packet both in time and along all coordinates. Each Fourier-decomposed wave would have to be propagated via the Kirchhoff's formula before recovering the spatio-temporal wave via an inverse Fourier transform. Such a procedure is used in numerical simulations with SRW reported below. A comparison between analytical theory and numerical results was found to be well within practically required accuracy.

${ }^{2}$ Taking into account that the depth of field is suppressed it also means that all rays have the same delay from travel between radiating and receiving points in the course of particle motion in the undulators.
}

\section{A. Small K parameter}

For the case when $K \ll 1$ Eq. (3) simplifies to

$$
E_{x}=\cos \left(\omega_{u} t^{\prime}\right) \frac{4 e \gamma^{4} \omega_{u} \theta_{e}}{c R} \frac{1+(\gamma \theta)^{2}\left[1-\cos ^{2}(\phi)\right]}{\left[1+(\gamma \theta)^{2}\right]^{3}} .
$$

Accounting that $\omega(\theta) \approx 2 \gamma^{2} \omega_{u} /\left(1+(\gamma \theta)^{2}\right)$ and $t\left(t^{\prime}\right) \approx$ $t^{\prime}\left(1+(\gamma \theta)^{2}\right) / 2 \gamma^{2}$ yields a straightforward evaluation of Eq. (6). Plugging the obtained expression into Eq. (5) noting that $\left|\boldsymbol{r}-\boldsymbol{r}^{\prime \prime}\right| \approx R_{O}$ and assuming a circular lens aperture so that $d \Sigma \approx R_{o}^{2} \theta d \theta d \phi$ we find the amplitude of the electric field at the center of the kicker

$$
E_{x}=\frac{4 e \gamma^{3} \omega_{u}^{2} K}{3 c^{2}} f_{L}\left(\gamma \theta_{m}\right),
$$

where $f_{L}(x)=1-1 /\left(1+x^{2}\right)^{3}$ describes the field reduction due to the finite angular acceptance, and $\theta_{m}$ is the angle subtended by the lens.

Since the depth of field has been suppressed by the large distance between the undulators and lens the amplitude of the electric field becomes constant over the length of the kicker. On axis the field has the dependence $\cos \left(\omega_{o} t-k_{o} z-\psi\right)$ where $\psi$ is determined by the difference in time of flight between the particle and its radiation. For small $K$ values $z \approx c t\left(1-1 / 2 \gamma^{2}\right)$ and hence the field seen by the electron is $E_{x} \cos \left(\omega_{u} t-\psi\right)$. Using $d \mathcal{E} / d t=$ $e v_{x} E_{x}$ yields the energy transfer to the particle

$$
\Delta \mathcal{E}=\frac{2}{3}\left(e \gamma K k_{u}\right)^{2} L_{u} f_{L}\left(\gamma \theta_{m}\right) \sin (\psi) .
$$

Figure 3 shows the relative kick strength as a function of angular acceptance of the focusing lens. We see that an acceptance of $\gamma \theta_{m}=0.8$ results in approximately $80 \%$

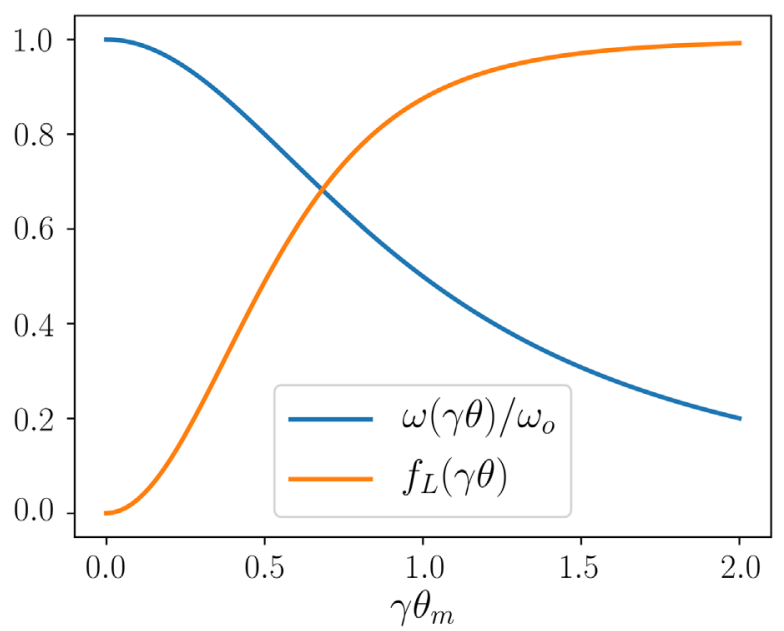

FIG. 3. The relative cooling rate as dependent on the angular acceptance of the focusing lens (orange trace) and ratio of forward to outermost angular frequency of the beam (blue trace). 
of the theoretical maximum kick. This corresponds to a relative bandwidth acceptance $\Delta \omega / \omega_{o}$ of $40 \%$. Here the bandwidth is determined by the instantaneous frequency of the wave and therefore is independent of the number of undulator periods. To obtain the actual bandwidth one has to account a finite number of undulator periods. For sufficiently large number of periods this additional spectrum widening can be neglected.

In the limit $\gamma \theta_{m} \gg 1$, Eq. (10) gives an energy transfer equal to the total amount of energy loss of the particle passing through both undulators in the absence of OSC, i.e., $\Delta \mathcal{E}=\Delta \mathcal{E}_{\text {tot }} \equiv \frac{2 L_{u}}{3}\left(e \gamma K k_{u}\right)^{2}$. If a particle is longitudinally displaced by a distance $s$ relative to the reference particle, in the course of its travel from pickup to kicker centers then its energy loss is modulated as

$$
\Delta \mathcal{E}(s)=\Delta \mathcal{E}_{\text {tot }}\left[1+\sqrt{G} f_{L}\left(\gamma \theta_{m}\right) \sin \left(k_{o} s\right)\right],
$$

where $k_{o}=2 \gamma^{2} \omega_{u} / c$ is the radiation wave number, $G$ the gain of optical amplifier (in power), and the additive 1 in the square brackets accounts for the energy loss in both undulators in the absence of interference, i.e., in the absence of OSC. The above expression neglects the finite length of the undulator-radiation pulse $L_{p} \approx N_{u} \lambda_{o}$ as typically $s$ is small fraction of $\lambda_{o}$. When this is not true the sinusoidal term in Eq. (11) should be multiplied by $\left(L_{p}-|s|\right) / L_{p}$ for $|s|<L_{p}$.

To find the transverse electric field distribution in the plane orthogonal to the axis and coming through the focal point we must retain the phase term in Eq. (5) accounting the phase advance correction. Let the observation point be $\boldsymbol{P}^{\prime \prime}=\left(R_{o}, \rho^{\prime \prime} \theta^{\prime \prime}, \rho^{\prime \prime} \phi^{\prime \prime}\right)$ then, Eq. (5) becomes

$$
\begin{aligned}
E_{x}\left(\rho^{\prime \prime}, \phi^{\prime \prime}\right)= & \frac{8 e \gamma^{6} \omega_{u}^{2} \theta_{e}}{c^{2}} \int_{0}^{2 \pi} \frac{d \phi}{2 \pi} \\
& \times \int_{0}^{\theta_{m}} \frac{1+(\gamma \theta)^{2}\left(1-2 \cos ^{2} \phi\right)}{\left(1+(\gamma \theta)^{2}\right)^{4}} \\
& \times \exp \left[\frac{i \rho^{\prime} k_{o} \theta \cos \left(\phi^{\prime \prime}-\phi\right)}{1+(\gamma \theta)^{2}}\right] \theta d \theta
\end{aligned}
$$

Performing the integration over $\phi$ one obtains

$$
\begin{aligned}
E_{x}\left(\rho^{\prime \prime}, \phi^{\prime \prime}\right)= & \frac{8 e \gamma^{6} \omega_{u}^{2} \theta_{e}}{c^{2}} \int_{0}^{\theta_{m}}\left\{J_{0}\left(\frac{\rho k_{o} \theta}{1+(\gamma \theta)^{2}}\right)\right. \\
& \left.+(\gamma \theta)^{2} J_{2}\left[\frac{\rho k_{o} \theta}{1+(\gamma \theta)^{2}} \cos \left(2 \phi^{\prime \prime}\right)\right]\right\} \frac{\theta d \theta}{\left[1+(\gamma \theta)^{2}\right]^{4}} .
\end{aligned}
$$

Figure 4 shows the relative field dependence in the transverse plane normalized to the limiting case when $\gamma \theta_{m}=\infty$. The point where the field reaches its first zero determines the half-size for the corresponding plane.

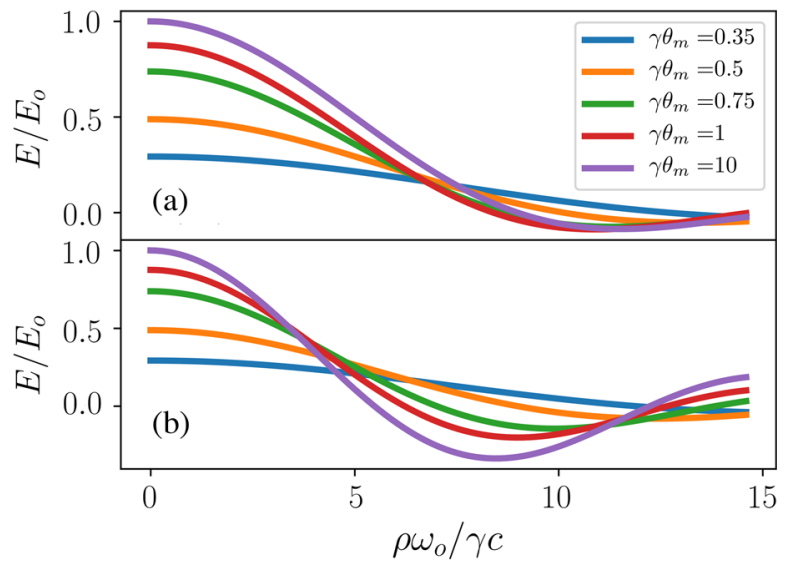

FIG. 4. Transverse dependence of the electric field at the focal point in the horizontal $\phi^{\prime \prime}=0$ (a) the vertical $\phi^{\prime \prime}=\pi / 2$ (b) plane. The field is normalized to its on-axis value $E_{0}$.

If $\gamma \theta_{m} \geq 0.1$ the horizontal and vertical half-sizes can respectively be estimated as

$$
\begin{aligned}
& x_{o} \approx \lambda_{o} \sqrt[3]{(1.51 \gamma)^{3}+\left(0.159+0.619 / \theta_{m}\right)^{3}} \text { and } \\
& y_{o} \approx \lambda_{o} \sqrt[3]{(1.08 \gamma)^{3}+\left(0.619 / \theta_{m}\right)^{3}}
\end{aligned}
$$

\section{B. Arbitrary $K$ value}

We now consider the case when $K$ takes an arbitrary value and restrict our attention to the on-axis field. In this case

$$
E_{x}=\frac{4 e \omega_{u}^{2} \gamma^{3} K}{3 c^{2}} F_{h}\left(K, \gamma \theta_{m}\right)
$$

where $0 \leq F_{h}\left(K, \gamma \theta_{m}\right) \leq 1$ is defined in Appendix and can be evaluated numerically. Integrating along the kicker as before but now also accounting for the longitudinal oscillations of the particle (see Appendix) yields the OSC kick

$$
\Delta \mathcal{E}=\frac{2 \pi}{3} e^{2} k_{o} N_{u} F_{T}\left(K, \gamma \theta_{m}\right)
$$

where $F_{T}\left(K, \gamma \theta_{m}\right)=K^{2}\left(1+K^{2} / 2\right) F_{h}\left(K, \gamma \theta_{m}\right) F_{u}(\kappa)$ is the dimensionless kick amplitude per undulator period. Figure 5 shows $F_{h}\left(K, \gamma \theta_{m}\right)$ and $F_{T}(K, \gamma)$ as a function of $K$ for various angular acceptances.

Next, we consider how these formulas are guiding the choice in undulator parameters. Equation (16) indicates that the kick amplitude increases with shorter wavelength. On the other hand, the wavelength must be chosen sufficiently long so that the high amplitude particles are not displaced too far in phase resulting in them being antidamped. For proton or heavy ion colliders, the cooling range should be about $5-\sigma$ or more of the beam equilibrium rms size. Thus 

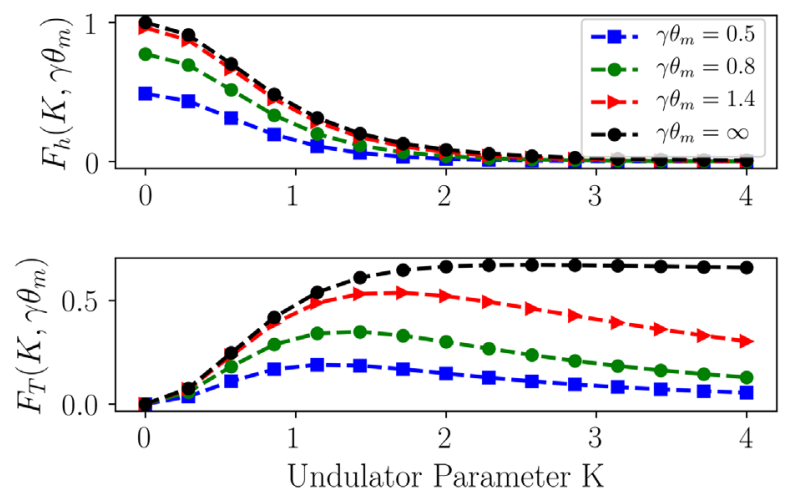

FIG. 5. Suppression factors $F_{h}\left(K, \gamma \theta_{m}\right)$ and $F_{T}\left(K, \gamma \theta_{m}\right)$ as a function of the undulator parameter $K$ for a range of lens angular acceptances.

the wavelength is chosen as short as possible while still satisfying the required cooling range. An additional constraint dictating the wavelength choice arise when considering the optical amplifier (OA). The addition of the OA introduces additional optical delay over passive OSC. At fixed wavelengths, the cooling range decreases inversely proportional with increasing optical delay [18] suggesting that OA gain media operating longer wavelengths are favored.

Using a telescope which corrects the depth-of-field effect (see Sec. III) makes the cooling force grow linear with undulator length and thus should be made as long as possible for the given allocated space for a cooling insertion. This leaves $K$ to be varied as a free parameter. From the bottom pane in Fig. 5 we see that for finite lens acceptances the growth of $F_{T}\left(K, \gamma \theta_{m}\right)$ saturates at moderate values of the undulator parameter $(K \leq 2)$. At larger $K$ values $F_{T}\left(K, \gamma \theta_{m}\right)$ begins to decrease but for a fixed undulator length, this reduction is offset by an increased number of periods leading to a levelling off of the kick amplitude for large $K$.

For a proof-of-principle demonstration of the OSC at IOTA [19] a large $K$ has the detrimental effect of spoiling the equilibrium beam emittance (prior to cooling) since dispersion at both the pickup and kicker is needed for horizontal cooling. Low equilibrium emittance is desirable as it enables OSC to be performed at a short wavelength while still having acceptable cooling ranges and a

TABLE II. Obtained values for OSC using the undulator parameters from Table I.

\begin{tabular}{lcl}
\hline \hline Parameter, symbol & Value & Units \\
\hline Peak electric field, $E_{x}$ & 11.8 & $\mathrm{~V} / \mathrm{m}$ \\
Suppresion factor, $F_{h}(1.03,0.8)$ & 0.24 & $\ldots$ \\
Period-normalized kick, $F_{u}(0.17)$ & 0.91 & $\ldots$ \\
Maximum energy exchange, $\Delta \mathcal{E}(\max )$ & 22 & $\mathrm{meV}$ \\
Electric field half-sizes, $x_{o} / y_{o}$ & $680 / 520$ & $\mu \mathrm{m}$ \\
\hline \hline
\end{tabular}

reasonable optical delay to accommodate an OA. Based on these considerations a value of $K=1.03$ was selected for the OSC experiment in IOTA. Table II compiles values related to the electric field and kick amplitude for the undulator parameters given in Table I.

\section{OPTICAL IMAGING WITH A TELESCOPE}

The above results were obtained for the condition that $R_{o} \gg L_{u}$. Under this assumption light emitted at a specific longitudinal location in the pickup is nominally refocused to the corresponding location in the kicker, and thus the field amplitude may be considered constant along the length of the kicker.

In an accelerator, the condition $R_{o} \gg L_{u}$ cannot be practically achieved. Instead, an imaging system with a transfer matrix $\mathbf{M}_{T}$ from pickup to kicker centers equal to $\pm \mathbf{I}$, where $\mathbf{I}$ is the identity matrix, is used. In this case, the transfer matrix between emitting and receiving points is $\mathbf{O}(l) \mathbf{M}_{T} \mathbf{O}(-l)= \pm \mathbf{I}$ where $\mathbf{O}(l)$ is the transfer matrix of a drift and $l$ is a displacement measured from pickup/kicker center.

The simplest telescope for the $+\mathbf{I}$ case consists of three lenses with one located at the midpoint of the optical transport (lens 2) and two identical lenses located on each side (referred to as lenses 1 ). The focal lengths of the lenses 1 and 2 are respectively given by

$$
F_{1}=\frac{L_{1} L_{2}}{L_{1}+L_{2}} \quad F_{2}=\frac{L_{2}^{2}}{2\left(L_{1}+L_{2}\right)}
$$

where $2\left(L_{1}+L_{2}\right)$ is the distance from pickup to kicker centers, $F_{2}$ is placed at the midpoint of the cooling insertion and the $F_{1}$ lenses are placed on both sides of $F_{2}$ at a distance $L_{2}$ away. In the case of the transfer matrix equal to $-\mathbf{I}$ we have

$$
F_{1}=L_{2} \quad F_{2}=-\frac{L_{2}^{2}}{2\left(L_{1}-L_{2}\right)}
$$

For a passive OSC test in IOTA the -I telescope matrix is chosen $^{3}$ and the lens focal lengths and positions are given in Table III.

For the active test, the telescope has the additional requirement of tight focusing of the pickup radiation in the amplifier. For this case, the $+\mathbf{I}$ telescope must be used. In passing, we note that by setting $L_{1}=L_{2}$ in Eq. (18) the center lens can be eliminated. This telescope would be desirable to use as the weaker focusing lenses would reduce

\footnotetext{
${ }^{3}$ Such choice is supported by smaller focusing chromaticity and also results in smaller transverse separation between radiation and particle in the kicker undulator. For chosen IOTA beam optics this condition is fully satisfied for horizontal plane while only partially for the vertical plane.
} 
TABLE III. Geometrical parameters of lens telescope for the passive-OSC proof-of-principle experiment in IOTA.

\begin{tabular}{lccl}
\hline \hline Parameter & Symbol & Value & Units \\
\hline Distance from kicker to lens 1 & $L_{1}$ & 143 & $\mathrm{~cm}$ \\
Distance from lens 1 to lens 2 & $L_{2}$ & 32 & $\mathrm{~cm}$ \\
Focal length of lens 1 & $F_{1}$ & 32 & $\mathrm{~cm}$ \\
Focal length of lens 2 & $F_{2}$ & 4.6 & $\mathrm{~cm}$ \\
\hline \hline
\end{tabular}

chromatic effects. It, however, cannot be used in IOTA as it would place the lenses in the path of the particle beam.

The performance of the telescope was studied using SRW [17]. As expected the telescope supports longitudinal point-to-point imaging along the kicker as evident from the time-domain waveform modulation. A reduction in field amplitude occurs at the edges of the kicker as the effective angular aperture is reduced for light emitted at the pickup edges. For parameters anticipated in IOTA this effect reduced the kick amplitude by $10 \%$.

Finally we note that due to a combination of over and underfocusing of the pickup radiation inside the kicker, simulations with SRW show that a single-lens can be just as effective as a telescope even for cases where $R_{o}$ is only a few times greater than $L_{u}$. For this case simulations showed no significant deviations from the expressions obtained above at the accuracy required to design an implementation of the OSC.

\section{FOCUSING ERRORS AND LENS CHROMATICITY}

We now consider two sources of errors: (1) manufacturing errors of the telescope lenses, and (2) lens chromaticity which plays a significant role due to the wide bandwidth required for the OSC. To simplify the problem we consider these errors separately.

\section{A. Focal length error}

We first consider the case of a single focusing lens and assume $K \ll 1$ in which case the electric field at the focus becomes:

$$
E_{x}=\frac{8 e \gamma^{5} \omega_{u}^{2} K}{c^{2}}\left|\int_{0}^{\theta_{m}} \exp [i \Phi(\theta)] \frac{\theta d \theta}{\left[1+(\gamma \theta)^{2}\right]^{4}}\right|
$$

where $\Phi(\theta)$ is an additional phase term accounting an error in focusing strength. The phase advance of light passing through the lens will be given by $k(\theta) \rho^{2} /[2(F+\delta f)]$. Then since, at the lens surface, $\rho=2 F \theta$ and assuming $\delta F \ll F$ we find $\Phi(\theta)=-2 \theta^{2} k_{o} \delta F /\left[1+(\gamma \theta)^{2}\right]$. Figure 6 shows the relative reduction in the field at the focal point as a function of $\delta_{F}$ for a variety telescope angular acceptances. Taking the case of $\gamma \theta_{m}=0.8$ and requiring the focusing errors not to reduce the field by more than $2 \%$ requires $\delta F<1.23 \mathrm{~cm}$.

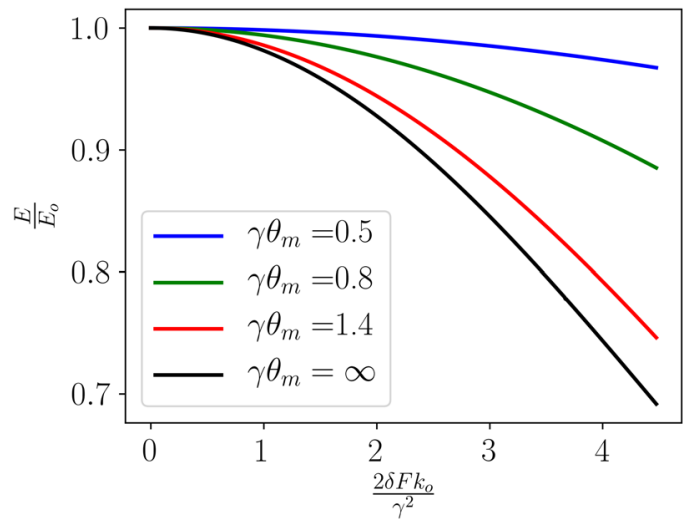

FIG. 6. Relative reduction of the on-axis electric field at the focal point as a function of the dimensionless focusing error and for different angular acceptances of lens telescope.

To find tolerances of the focusing errors of the three-lens telescope we, first, consider a single lens telescope. A focusing error of $\delta F$ results in a displacement of focal point $\delta s=4 \delta F$. Then, we require that a focusing error for a single lens of the telescope results in the same displacement of the focal point as in the single lens case. Introducing relative errors of telescope lenses $\left(f_{k} \equiv-\delta F_{k} / F_{k}, k=1\right.$, $2,3)$ multiplying the corresponding matrices and leaving only the first order terms we obtain for the $M_{12}$ transfer matrix element:

$$
M_{12}=-2\left(L_{1}-L_{2}\right) f_{2}+\frac{L_{1}^{2}}{L_{2}}\left(f_{1}+f_{3}\right) .
$$

The focal point displacement is equal to $-M_{12} / M_{22}$. Accounting that the matrix is close to identity matrix $\left(\left|M_{22}\right| \approx 1\right)$ one obtains $\delta s \approx M_{12}$. Considering only one focal length error at a time, we find that for the telescope parameters presented in Table III that the fractional error on the focal length value should be within $\left|f_{1}\right|=\left|f_{3}\right| \leq 0.8 \%$ and $\left|f_{2}\right| \leq 2.3 \%$. Performing similar calculations for the displacement of the outer lenses one obtains $\left|\delta L_{2}\right| \leq$ $2.3 \mathrm{~mm}$. Note that longitudinal displacement of the entire telescope results in no error because of its point-to-point imaging property.

\section{B. Lens chromaticity}

One of the main challenges with the optical system comes from the large relative bandwidth $\sim 30 \%$ associated to the radiation; see Fig. 2. Chromaticity within the lenses results in misfocusing of the radiation in the kicker. The lens focal length depends on wavelength as $F=$ $F_{o}\left(n_{\text {opt }}-1\right) /(n(\lambda)-1)$ where $n_{\text {opt }}$ is index of refraction at $\lambda_{\text {opt }}$, the wavelength that minimizes the telescope focusing errors. In the case of the OSC experiment at IOTA, Barium fluoride $\left(\mathrm{BaF}_{2}\right)$ was selected for the lens material owing to its low chromaticity over the OSC bandwidth of 
$[2.2,3.2] \mu \mathrm{m}$. The wavelength dependence of the index of refraction is described by the Sellmeier's equation [20]

$$
\begin{aligned}
n(\lambda)^{2}= & 1.33973+\frac{0.81070 \lambda^{2}}{\lambda^{2}-0.10065^{2}} \\
& +\frac{0.19652 \lambda^{2}}{\lambda^{2}-29.87^{2}}+\frac{4.52469 \lambda^{2}}{\lambda^{2}-53.82^{2}} .
\end{aligned}
$$

For $\lambda=2.2 \mu \mathrm{m}$ we have $d n / d \lambda=-3.21 \times 10^{-3} \mu \mathrm{m}^{-1}$ and a group-velocity dispersion (GVD) value of $-9.7405 \mathrm{fs}^{2} / \mathrm{mm}$. The optical-transport transfer matrix from pickup to kicker can now be computed as a function of wavelength and the displacement of the focal point as a function of the wavelength can be inferred using $\delta s(\lambda)=-M_{12}(\lambda) / M_{22}(\lambda)$. Intuitively $\lambda_{\text {opt }}$ will be somewhere in the middle of the OSC band where the photon flux and the growth rate of the kick amplitude with respect to lens angular acceptance are largest. This leads us to choose $\lambda_{\text {opt }}=2.6 \mu \mathrm{m}$. Computation of $\delta s(\lambda)$ results in a focal displacement ranging from 3 to $-4 \mathrm{~cm}$ within the interval $\lambda \in[2.2,3.2] \mu \mathrm{m}$. The increase of the total spot size at the focal point is estimated as

$$
r(\lambda)=\sqrt{\frac{x_{o}^{2}+y_{o}^{2}}{2}+\left(\theta_{m} \delta s(\lambda)\right)^{2}},
$$

where $x_{o}$ and $y_{o}$ were computed using Eq. (14). It results in a $2 \%$ and $4 \%$ increase in the spot size at 2.2 and $3.2 \mu \mathrm{m}$. As a first order approximation the field (and hence the kick amplitude) is reduced as $1 / r(\lambda)$ and thus we expect focusing chromaticity to reduce the OSC kick by at most of few percent in case of IOTA.

To confirm our choice of $\lambda_{\text {opt }}$ the on-axis field was computed with SRW for a range of design wavelengths for
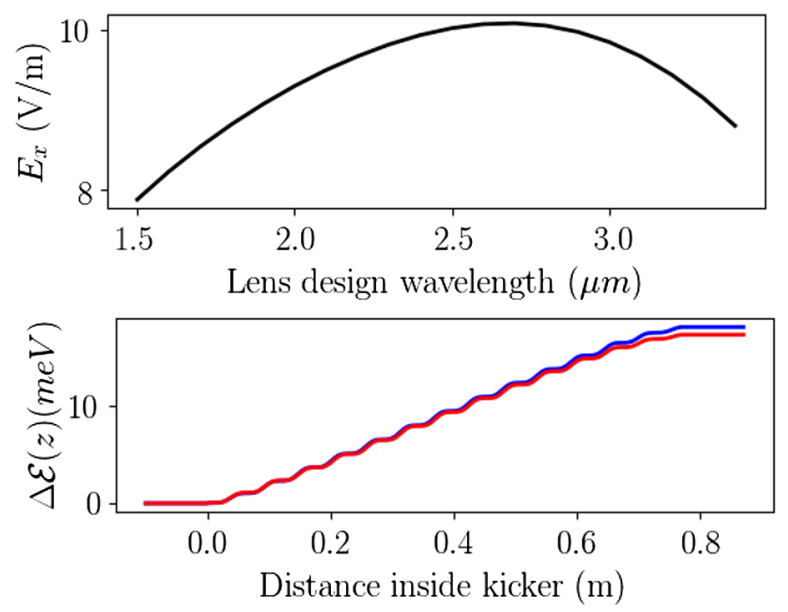

FIG. 7. Top: The electric field amplitude at the kicker center for different design wavelengths of the telescope lenses. Bottom: The energy exchange for a particle copropagating with the simulated radiation field from SRW through the kicker with (red trace) and without (blue trace) accounting for the lens dispersion. the lenses. Since SRW computations are done in the Fourier domain it is straightforward to account for lens dispersion. The top panel in Fig. 7 shows the resulting dependence of electric-field amplitude on the design wavelength of the telescope. The bottom pane displays the energy gain for an electron with its arrival phase chosen to be $\psi=\pi / 2$ with and without accounting for dispersion. Including dispersive effects decreases the kick by approximately $4 \%$. The SRW simulatiozns also include pulse broadening (reduction in field amplitude) from the second-order dispersion.

\section{CONCLUSION}

The presented semianalytic approach allows one to compute the energy kick a particle receives in a single pass through an OSC insertion. It provides a straightforward way of determining the main undulator and optical telescope parameters and tolerances. Above we mostly considered a passive scheme for the OSC which is suitable only for a proof-of-principal demonstration with electrons. For cooling of hadrons an amplifier providing 20-30 dB within a single pass is required. As a first order estimate the kick amplitude is proportional to $\sqrt{G}$ of the pickup radiation gain in power. A further refinement that accounts the finite amplifier bandwidth (and possibly) additional 2nd order dispersion will reduce the overall kick value. These effects are easily accounted with SRW. For example in an active test of the OSC in IOTA an amplifier, based on $\mathrm{Cr}: \mathrm{ZnSe}$, gives $7 \mathrm{~dB}$ of gain. However the kick amplitude is expected to increase only by a factor 1.65 when host dispersion and amplifier bandwidth are taken into account [21].

Throughout our analysis, we have considered radiation emitted by a single particle. However, in practice, a given particle will be affected by the radiation emitted by its neighboring particles in addition to its own. Such an interparticle interaction will result in a "heating" effect which is straightforward to describe using the theory developed for conventional stochastic cooling [1]. Neglecting this heating effect in the present analysis is justified as the considered proof-of-principle experiment at the IOTA ring involves passive OSC or low-gain amplification and a low number of particle in the OSC sampling slice.

The model presented in this paper was implemented in the ELEGANT [22] program to successfully verify, via particle tracking, the main features of OSC cooling for the experiment planned at IOTA [23]. We finally note that the formalism presented in this paper is general and could be extended to other OSC configurations. It could additionally prove useful in other electron-radiation interaction mechanisms involving the transport and manipulation of radiation from undulator magnets.

\section{ACKNOWLEDGMENTS}

The authors would like to express their gratitude to J. Ruan and A. Romanov from Fermilab. The work of 
M. A. and P. P. was supported by the U.S. Department of Energy under Contract No. DE-SC0013761 to Northern Illinois University. Fermilab is managed by the Fermi Research Alliance, LLC for the U.S. Department of Energy Office of Science Contract No. DE-AC0207CH11359.

\section{APPENDIX: ELECTRIC FIELD AND KICK AMPLITUDE FOR ARBITRARY VALUES OF $K$}

In this Appendix we derive an expression for the electric field and kick amplitude for arbitrary values of $K$. We begin with Eq. (6) with the integration variable changed from emitter to observer time

$$
E_{\omega}(\theta, \phi)=\frac{\omega(\theta)}{\pi} \int_{0}^{\frac{2 \pi}{\omega_{u}}} E_{x}\left(t^{\prime}\right) \exp \left(-i \omega(\theta) t\left(t^{\prime}\right)\right) \frac{d t}{d t^{\prime}} d t^{\prime}
$$

Insertion of Eq. (3), Eq. (7) and its derivative into the above equation and performing some simplifications yields

$$
E_{\omega}(\theta, \phi)=\frac{4 \gamma^{4} e \omega_{u}^{2} \theta_{e}}{\pi c R_{o}} \int_{0}^{2 \pi}\left[F_{c}\left(\Theta, K, \tau^{\prime}, \phi\right) \times \frac{1+\Theta\left(1-2 \cos ^{2}(\phi)\right)-2 \Theta K \cos (\phi) \sin \left(\tau^{\prime}\right)-K^{2} \sin ^{2}\left(\tau^{\prime}\right)}{\left[1+\Theta^{2}+2 \Theta K \cos (\phi) \sin \left(\tau^{\prime}\right)+K^{2} \sin ^{2}\left(\tau^{\prime}\right)\right]^{3}}\right] d \tau^{\prime}
$$

where

$F_{c}\left(\Theta, K, \tau^{\prime}, \phi\right)=\cos \left(\tau^{\prime}\right) \times \exp \left[-i \tau^{\prime}+i \frac{K^{2} \sin \left(2 \tau^{\prime}\right)+8 \Theta K \cos (\phi) \cos \left(\tau^{\prime}\right)}{4\left(1+\Theta^{2}+K^{2} / 2\right)}\right] \times\left[1+\frac{4 \Theta K \cos (\phi) \sin \left(\tau^{\prime}\right)-K^{2} \cos \left(2 \tau^{\prime}\right)}{2\left(1+\Theta^{2}+K^{2} / 2\right)}\right]$

and the variables $\Theta=\theta \gamma$ and $\tau^{\prime}=\omega_{u} t^{\prime}$ were introduced. This expression can then be inserted into Eq. (5) which will yield the result

$$
E_{x}=\frac{4 \gamma^{3} e \omega_{u}^{2} K}{3 c^{2}} F_{h}(K, \gamma \theta)
$$

where

$$
F_{h}(K, \gamma \theta)=\frac{3}{\pi^{2}} \int_{0}^{\Theta_{m}} \int_{0}^{2 \pi} \int_{0}^{2 \pi}\left[\frac{\Theta F_{c}\left(\Theta, K, \tau^{\prime}, \phi\right)}{1+\Theta^{2}+K^{2} / 2} \times \frac{1+\Theta\left[1-2 \cos ^{2}(\phi)\right]-2 \Theta K \cos (\phi) \sin \left(\tau^{\prime}\right)-K^{2} \sin ^{2}\left(\tau^{\prime}\right)}{\left[1+\Theta^{2}+2 \Theta K \cos (\phi) \sin \left(\tau^{\prime}\right)+K^{2} \sin ^{2}\left(\tau^{\prime}\right)\right]^{3}}\right] d \Theta d \phi d \tau^{\prime}
$$

Next to find the kick we integrate the energy exchange between the particle and field. For a finite $K$ value, in addition to its transverse motion, the particle also oscillates longitudinally. This will lead to an efficiency factor $F_{u}(\kappa) \leq 1$ that must be included in computation of the kick amplitude. As before we assume the electric field amplitude seen by the electron stays constant as it propagates along the kicker. Let the field be described as $E=E_{x} \cos \left(\omega_{o} t-k_{o} z\right)$. Integration of $v_{z}$ given in Eq. (2) gives

$$
z=c\left[\left(1-\frac{1}{2 \gamma^{2}}-\frac{\theta_{e}^{2}}{4}\right) t+\frac{\theta_{e}^{2}}{8 \omega_{u}} \sin \left(2 \omega_{u} t\right)+z_{o}\right]
$$

Inserting this into the expression for the field, recalling that $\omega_{u}=\omega_{o}\left(1 / 2 \gamma^{2}+\theta_{e}^{2} / 4\right)=\omega_{o}\left(1+K^{2} / 2\right) / 2 \gamma^{2}$ and defining $\kappa=\theta_{e}^{2} \omega_{o} / 8 \omega_{u}=K^{2} /\left(4+2 K^{2}\right)$ yields:

$$
E=E_{x} \cos \left[\omega_{u} t-\kappa \sin \left(2 \omega_{u} t\right)-\psi\right] .
$$

Then using $d \mathcal{E} / d t=e v_{x} E_{x}$ gives

$$
\Delta \mathcal{E}=\frac{e c K E_{x}}{\gamma} \int_{0}^{\frac{2 \pi N_{u}}{\omega_{u}}} \cos \left[\omega_{u} t-\kappa \sin \left(2 \omega_{u} t\right)-\psi\right] \sin \left(\omega_{u} t\right) d t .
$$

The above integral is easily evaluated with the substitution $\xi=\omega_{u} t$ and the aid of the following two identities

$$
\begin{aligned}
& \int_{0}^{2 \pi} \cos (\xi-\kappa \sin 2 \xi) \sin \xi d \xi \\
& \quad=0 \int_{0}^{2 \pi} \sin (\xi-\kappa \sin 2 \xi) \sin \xi d \xi=\pi\left[J_{0}(\xi)-J_{1}(\xi)\right]
\end{aligned}
$$

which yields

$$
\Delta \mathcal{E}=\frac{e K E_{x} L_{u}}{2 \gamma} \sin (\psi) F_{u}(k)
$$


where

$$
F_{u}(\kappa)=J_{0}(\kappa)-J_{1}(\kappa) .
$$

Thus accounting for the longitudinal motion of the particle reduces the kick by a factor $F_{u}(\kappa)$.

So far all expressions apply to a planar undulator. For a helical undulator there are both horizontal and vertical electric field polarizations and so Eq. (A4) is modified as

$$
E_{i}=\frac{4 \gamma^{3} e \omega_{u}^{2} K}{3 c^{2}} F_{h h}(K, \gamma \theta)
$$

with $i=x, y$ and

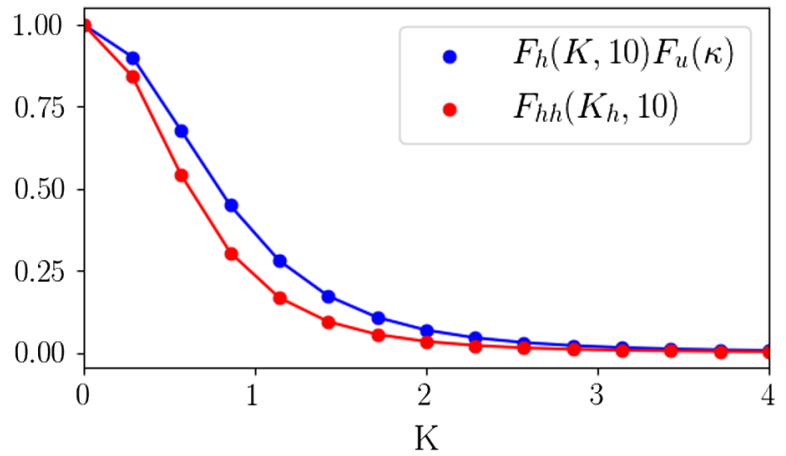

FIG. 8. Suppression factors for the planar and helical undulators with $\gamma \theta_{m}=10$.

$F_{h h}\left(K_{h}, \gamma \theta\right)=\frac{3}{\pi} \int_{0}^{\theta_{m}} \Theta \int_{0}^{2 \pi} \frac{1+\Theta^{2}+K_{h}^{2}+2 \Theta K_{h} \sin (\tau)-2\left[\Theta^{2} \exp (-i \tau)-i \Theta K_{h}\right] \cos (\tau)}{\left(1+\Theta^{2}+K_{h}^{2}\right)^{2}\left[1+\Theta^{2}+K_{h}^{2}+2 \Theta K_{h} \sin (\tau)\right]^{2}} \exp \left(\frac{2 i \Theta K_{h} \cos (\tau)}{1+\Theta^{2}+K_{h}^{2}}\right) d \tau d \Theta$.

where $K_{h}=\sqrt{2} K$ is the helical undulator parameter and it is assumed that the vertical and horizontal magnetic field components are of the same magnitude and $\pi / 2$ out of phase. In this case there are no oscillations in the longitudinal directions so that $F_{u h}(\kappa)=1$ for all $K$. Replacing $K$ and $F_{h}\left(K, \gamma \theta_{m}\right)$ with $K_{h}$ and $F_{h h}\left(K, \gamma \theta_{m}\right)$ in Eq. (16) yields the kick amplitude for the helical undulator.

The suppression factors for the planar and helical undulator are plotted in Fig. 8. In the limit of $K \ll 1$ the kick amplitude is proportional to $K^{2}$ and so a helical undulator yields twice the kick over a planar undulator. However with increasing $K$ the efficiency of the helical undulator decreases due to the emitted radiation being directed more off-center, while most of the radiation is admitted forward for a planar unudulator. This effect reduces the advantage of the helical undulator for large $K$ values. Thus the choice in undulator type should be determined by accounting all practical limitations such as: allocated length, wavelength of radiation, and achievable $K$ value.

[1] D. Möhl, G. Petrucci, L. Thorndahl, and S. van der Meer, Physics Reports Physics and technique of stochastic cooling, Phys. Rep. 58, 73 (1980).

[2] S. van der Meer, CERN Internal Report No. CERN ISR-PO 72/31, 1970.

[3] P. Bramham, G. Carron, H. G. Hereward, K. Hubner, W. Schnell, and L. Thorndahl, Stochastic cooling of a stored proton beam, Nucl. Instrum. Methods Phys. Res. 125, 201 (1975).

[4] A. A. Mikhailichkenko and M. S. Zolotorev, Optical Stochastic Cooling, Phys. Rev. Lett. 71, 4146 (1993).

[5] M. S. Zolotorev and A. A. Zholents, Transit-time method of optical stochastic cooling, Phys. Rev. E 50, 3087 (1994).
[6] S. Y. Lee, Y. Zang, and K. Y. Ng, Damping dynamics of optical stochastic cooling, Nucl. Instrum. Methods Phys. Res. 532, 340 (2004).

[7] M. Blaskiewicz, Cooling of high-energy hadron beams, Annu. Rev. Nucl. Part. Sci. 64, 299 (2014).

[8] M. Babzien, I. Ben-Zvi, I. Pavlishin, I. V. Pogorelsky, V. E. Yakimenko, A. A. Zholents, and M. S. Zolotorev, Optical stochastic cooling for RHIC using optical parametric amplification, Phys. Rev. ST Accel. Beams 7, 012801 (2004).

[9] A. Zholents, M. Zolotorev, and W. Wan, Optical stochastic cooling of muons, Phys. Rev. ST Accel. Beams 4, 031001 (2001).

[10] V. Lebedev, Test of Optical Stochastic Cooling in Fermilab, in Proceedings of the 52nd ICFA Advanced Beam Dynamics Workshop on High-Intensity and High-Brightness Hadron Beams (HB2012), Beijing, China, 2012.

[11] V. Lebedev, Yu. Tokpanov, and M. Zolotorev, in Proceedings of the 25th Particle Accelerator Conference, PAC2013, Pasadena, CA, 2013 (IEEE, New York, 2013), p. 422.

[12] E. G. Bessonov, M. V. Gorbunkov, A. A. Mikhailichenko, A. L. Osipov, and A. V. Vinogradov, Nuovo Cimento Soc. Ital. Fis. C034N4, 93 (2011).

[13] S. Antipov et al., IOTA (Integrable Optics Test Accelerator): Facility and experimental beam physics program, J. Instrum. 12, T03002 (2017).

[14] O. Chubar and P. Elleaume, in Proceedings of the 6th European Particle Accelerator Conference, Stockholm, 1998 (IOP, London, 1998), p. 1177.

[15] O. Chubar, P. Elleaume, S. Kuznetsov, and A. Snigirev, X-Ray FEL optics and instrumentation, Proceedings of SPIE 4769, Optical Design and Analysis Software II, 2002, (2001).

[16] O. Chubar et al., in Proceedings of the 29th Free Electron Laser Conference, Novosibirsk, Russia (BINP, Novosibirsk, 2007), p. 192.

[17] M. Andorf, V. A. Lebedev, P. Piot, and J. Ruan, Waveoptics modeling of the optical-transport line for passive 
optical stochastic cooling, Nucl. Instrum. Methods Phys. Res., Sect. A 883, 166 (2018).

[18] V. A. Lebedev, Optical stochastic cooling, in ICFA Beam Dynamics Newsletter (2014), pp. 100-116.

[19] V. Lebedev and A. L. Romanov, Optical stochastic cooling at the IOTA ring, Proceedings of COOL2015, Newport News VA (2015), p. 123-127.

[20] H. H. Li, Refractive index of alkaline earth halides and its wavelength and temperature derivatives, J. Phys. Chem. Ref. Data 9, 161 (1980).
[21] M. B. Andorf, V. A. Lebedev, P. Piot, and J. Ruan, Amplifier for Optical Stochastic Cooling, in Conference on Lasers and Electro-Optics, OSA Technical Digest (online) (Optical Society of America, 2017), Paper JW2A.90.

[22] M. Borland, ELEGANT: A flexible SDDS-compliant code for accelerator simulation, Advanced Photon Source LS-287, 2000.

[23] M. B. Andorf, P. Piot, and V. A. Lebedev, in Proceedings of 2018 International Particle Accelerator Conference (IPAC18), Vancouver BC (2018). 